\title{
OCLC begrüßt neue WMS-Anwender in der Community
}

\section{Neue WorldShare ${ }^{\circledR}$ Management Services-Installationen in Deutschland und der Schweiz}

https://doi.org/10.1515/bd-2021-0088

Mehrere Bibliotheken in Deutschland und der Schweiz haben sich kürzlich für die WorldShare ${ }^{\circledR}$ Management Services (WMS) von OCLC als neues, cloud-basiertes Bibliotheksmanagementsystem entschieden.

WorldShare ${ }^{\circledR}$ Management Services (WMS) ist ein cloud-basiertes Bibliotheksmanagementsystem mit WorldCat als Basis, das alle Arbeitsabläufe einer Bibliothek abdeckt und es Bibliotheksmitarbeiter*innen ermöglicht, das gemeinsame Datennetzwerk und die Technologie von OCLC für effizientere Arbeitsabläufe zu nutzen. Mit WMS erhalten Bibliotheksnutzer^innen einen erweiterten Zugang zu Bibliotheksbeständen aller Art weltweit.

$\mathrm{Zu}$ den Bibliotheken, die sich der WMS-Community angeschlossen haben, gehören u.a.:

- Franklin University - eine Internationale Universität in der südschweizerischen Stadt Lugano. Sie gehört zu AMICAL (American International Consortium of Academic Libraries), einer Vereinigung von 29 Universitäten in 22 Ländern.

- Hochschule der Polizei Brandenburg - auf dem modernen Campus der Hochschule in Oranienburg werden die Beamtinnen und Beamten des mittleren und des gehobenen Polizeivollzugsdienstes des Landes Brandenburg ausgebildet.

- Kühne Logistics University (KLU) - eine private, staatliche anerkannte wissenschaftliche Hochschule für Logistik und Unternehmensführung mit Sitz in Hamburg. Träger der Hochschule ist die Kühne Stiftung. Die KLU forscht und lehrt in den Schwerpunktbereichen Logistik, Supply Chain Management und Management. Eine direkte Anbindung an den K10plus ist geplant.

- Helmholtz-Zentrum für Umweltforschung (UFZ), Leipzig - Das UFZ hat sich mit seinen derzeit 1.100 Mitarbeiterinnen und Mitarbeitern als internationales Kompetenzzentrum für Umweltwissenschaften einen hervorragenden Ruf erworben. Es ist Teil der größten Wissenschaftsorganisation Deutschlands der Helmholtz-Gemeinschaft. Für eine Balance zwischen gesellschaftlicher 
Entwicklung und langfristigem Schutz unserer Lebensgrundlagen im Sinne einer nachhaltigen Entwicklung $\mathrm{zu}$ sorgen, steht im Mittelpunkt der Forschung.

Diese Institutionen setzen auf WorldShare ${ }^{\circledR}$ Management Services (WMS) als umfassendes, hochmodernes und cloud-basiertes Bibliotheksmanagementsystem, um ihre elektronischen, digitalen und gedruckten Bibliotheksbestände effizient und unter einer Oberfläche zu verwalten. Eine Single-Search Suche im Bestand und weiteren Datenquellen wie u. a. auch Open Access-Ressourcen unterstützen Studierende und Bibliotheksbenutzer*innen.

Insbesondere für die in Verbünden arbeitenden Bibliotheken ist der nahtlose Datenaustausch und die Anbindung an die jeweilige Verbunddatenbank (hebis Verbunddatenbank, K10plus, B3Kat) wichtig. Dieser ist durch entsprechende Schnittstellen und Echtzeit-Synchronisation mit WMS gewährleistet.

Andreas Schmidt, General Manager bei OCLC, sagte dazu: „Unser cloud-basiertes Bibliotheksmanagementsystem wird immer beliebter. Wir freuen uns, den genannten Bibliotheken mit den WorldShare ${ }^{\circledR}$ Management Services eine zukunftweisende Bibliothekslösung bieten zu können. Die Einbindung in bestehende regionale Bibliotheksinfrastrukturen ist dabei genauso wichtig wie die Anbindung an ein globales Netzwerk wie den OCLC WorldCat, mit 500 Millionen Titeln und über 3 Milliarden Bestandsnachweisen die größte bibliografische Datenbank der Welt.“

Mehr als 700 Bibliotheken weltweit nutzen inzwischen WorldShare ${ }^{\circledR}$ Management Services als Plattform für ihre Bibliotheksdienste.

Hier finden Sie weitere Informationen zu den WorldShare ${ }^{\circledR}$ Management Services: https://connect.oclc.org/de/wms?utm_campaign=de-wms-infohub-2020campaign\&utm_medium=email\&utm_source=microsoft-outlook\&utm_content= page-relaunch.

\section{Über OCLC}

OCLC ist ein gemeinnütziger, weltweit tätiger Bibliotheksverbund, der gemeinsam nutzbare technische Dienste, Lösungen und Gemeinschaftsprogramme bereitstellt, damit Bibliotheken die Bereiche Lernen, Forschung und Innovationen besser voranbringen können.

Über OCLC erstellen und pflegen die Mitgliedsbibliotheken WorldCat, das umfassendste globale Netzwerk von Daten zu Bibliothekssammlungen und -Services. Einen Effizienzgewinn für Bibliotheken verspricht WorldShare ${ }^{\circledR}$, ein vollständiges Paket mit Bibliotheksmanagementanwendungen und -Services, die auf einer offenen, cloud-basierten Plattform basieren. Über ihre Zusammenarbeit und das Teilen des gesammelten Wissens der Welt können die Bibliotheken Men- 
schen dabei helfen, Antworten zur Lösung ihrer Probleme zu finden. Zusammen als OCLC, gestalten die Mitgliedsbibliotheken, Mitarbeiter ${ }^{\star}$ innen und Partner den Wandel des Informationsverhaltens der Menschen mit.

OCLC, WorldCat, WorldCat.org und WorldShare ${ }^{\circledR}$ sind Marken und/oder Dienstleistungsmarken von OCLC, Inc. Produkt-, Dienst- und Unternehmensnamen von Drittanbietern sind Marken und/oder Dienstleistungsmarken ihrer jeweiligen Eigentümer.

\section{Bei Rückfragen:}

OCLC GmbH, Gabriele Wolberg

Tel.: + 49 (0) 89 61308-326 | E-Mail: Gabriele.Wolberg@oclc.org

URL: http://www.oclc.org | Twitter: @OCLC_DE 